\title{
Emergency rigid bronchoscopy in two lower social class patients with mirror like complete pulmonary malignant atelectasis
}

\author{
Umberto Caterino ${ }^{1}$, Dario Amore ${ }^{2}$, Maria Chiara Petagna ${ }^{3}$, Dino Casazza ${ }^{2}$, Roberto Marchese ${ }^{4}$ \\ ${ }^{1}$ Interventional Pulmonology Unit, V Monaldi Hospital, Naples; ${ }^{2}$ Department of Thoracic Surgery, V Monaldi Hospital, \\ Naples; ${ }^{3}$ Pulmonology Unit, AO Santobono Hospital, Naples; ${ }^{4}$ Interventional Pulmonology Unit, La Maddalena Cancer \\ Center, Palermo, Italy
}

\begin{abstract}
Several factors as cultural factors and social class other than biological and genetic factor can affect symptom perception in patients with malignant airway obstruction. Poor perception of dyspnoea can result in the delayed seeking of medical care so increase access to intensive care due to impeding respiratory failure. In patients issued from malignant airway obstruction, therapeutic bronchoscopy procedure can not affect the endotracheal extubation although immediate airway patency can be obtained.
\end{abstract}

Correspondence: Umberto Caterino, Interventional Pulmonology Unit, V Monaldi Hospital, Via Leonardo Bianchi 1, 80131 Naples, Italy. Tel.+39.081.7064141. E-mail: caterinou@yahoo.it

Contributions: All the authors made a substantive intellectual contribution. All the authors have read and approved the final version of the manuscript and agreed to be accountable for all aspects of the work.

Conflict of interest: The authors declare that they have no competing interests, and all authors confirm accuracy.

Availability of data and materials: All data underlying the findings are fully available.

Ethics approval and consent to participate: No ethical committee approval was required for this case report by the Department, because this article does not contain any studies with human participants or animals. Informed consent was obtained from the patient included in this study.

Consent for publication: The patients gave their written consent to use his personal data for the publication of this case report and any accompanying images.

Key words: Malignant central airway; lung cancer; rigid bronchoscopy.

Received for publication: 12 August 2020.

Accepted for publication: 10 November 2020.

${ }^{\circ}$ Copyright: the Author(s), 2021

Licensee PAGEPress, Italy

Monaldi Archives for Chest Disease 2021; 91:1564

doi: 10.4081/monaldi.2021.1564

This article is distributed under the terms of the Creative Commons Attribution Noncommercial License (by-nc 4.0) which permits any noncommercial use, distribution, and reproduction in any medium, provided the original author(s) and source are credited.
We reported the outcome of two patients from lower social classes admitted in intensive care and underwent emergency rigid bronchoscopy for malignant complete pulmonary atelectasis.

\section{Introduction}

Unilateral pulmonary atelectasis can result from undetected and slowing progression of lung cancer in central airway obstruction. Lung cancer is a disease of high symptom burden, major psychosocial impact and poor prognosis [1]. Although diagnosis is individual, each patient operates within a social context that may influence negatively or positively to health-care process. Cultural factors and low social class can influence the communication between physicians and patient resulting in underestimated symptom intensities [2]. In malignant airway obstruction, the misperception of dyspnoea reveals a low alarm of life-threatening status with delayed seeking of medical care and increasing access to intensive care.

Therapeutic rigid bronchoscopy is often mandatory to restore central airway patency when critically distress respiratory making clear although the success of this bronchoscopic procedure can not affect the endotracheal extubation in patients undergoing mechanical ventilation [3].

We reported the outcome of two lower-class patients admitted in intensive care for malignant airway obstruction and underwent emergency rigid bronchoscopy to restore airways patency from complete unilateral pulmonary atelectasis.

\section{Case Report}

\section{Case \#1}

A 51-year-old patient, heavy current smoker, was admitted in intensive care for acute respiratory failure. The clinical history was previously laryngeal cancer, and the patient underwent radiotherapy many years ago. The relatives referred persistent dry cough, body weight loss and mild dyspnoea over the last months. No blood exam or CXR was performed before admission in emergency unit care where it was needed endotracheal intubation. The poor social class allowed underestimate the respiratory symptoms. At admission blood gas analysis showed $\mathrm{PaO}_{2} 38 \mathrm{mmHg}$ and $\mathrm{PaCO}_{2} 40 \mathrm{mmHg}$ and chest X-ray showed complete atelectasis of right lung (Figure 1a). A flexible bronchoscope was introduced through endotracheal tube and intraluminal mass obstructing main right lobe was detected (Figure 1b). 
Emergency rigid bronchoscopy and debulking of intraluminal mass allowed to re-establish the airway patency to the lower right bronchus replaced to tumour (Figure 1c). A neodymiumyttrium aluminium garnet (Nd-YAG) laser (20 Watt) was used to ablate the residual endobronchial tumour. A chest X-ray taken after debulking showed the re-expansion of right lung with atelectasis of lower right lobe (Figure 1d). Histological examination revealed lung squamous cell carcinoma. The patient died a week later due to difficulty weaning from the mechanical ventilator.

\section{Case \#2}

A 67-year-old patient with lung cancer was admitted to the intensive care unit and underwent emergency endotracheal intubation due to acute respiratory failure. The patient was lost to followup after chemotherapy because of poor socio-economic status and no significant symptoms were referred by relatives. At the admission, the blood gas analysis showed $\mathrm{PaO}_{2} 34 \mathrm{mmHg}$ and $\mathrm{PaCO}_{2} 40$ $\mathrm{mmHg}$ and chest $\mathrm{x}$ ray showed complete atelectasis of left lung (Figure 2a). Emergency rigid bronchoscopy was performed and endoluminal vegetation occluding left main bronchus and protruding into right main bronchus was observed (Figure 2b). Surprisingly an endoluminal vegetation was observed into distal left main bronchus after tumour resection (Figure 2c). Finally, mechanical debulking-laser assisted rigid bronchoscopy was performed and the patient was subsequently extubated after complete patency of left airway system (Figure $2 \mathrm{~d}$-e).

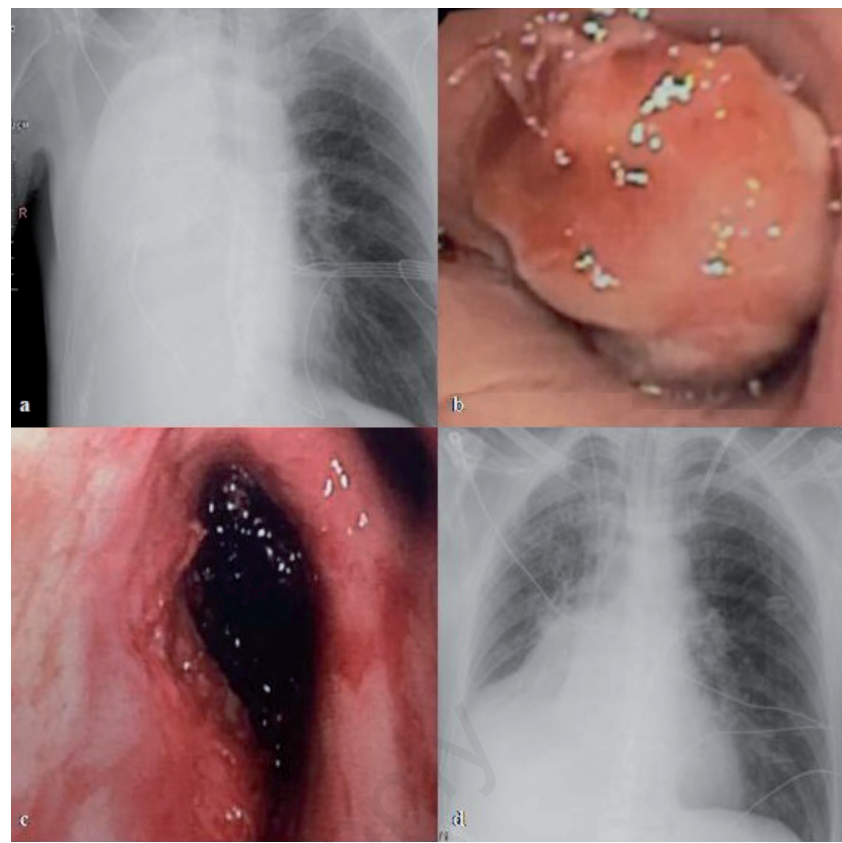

Figure 1. a) Chest X-ray at admission showed atelectasis of the right lung. b) Bronchoscopic view revealed endoluminal vascularized vegetation obstructing right main bronchus. c-d) Residual tumour invasion in lower bronchus lobe with distal atelectasis.

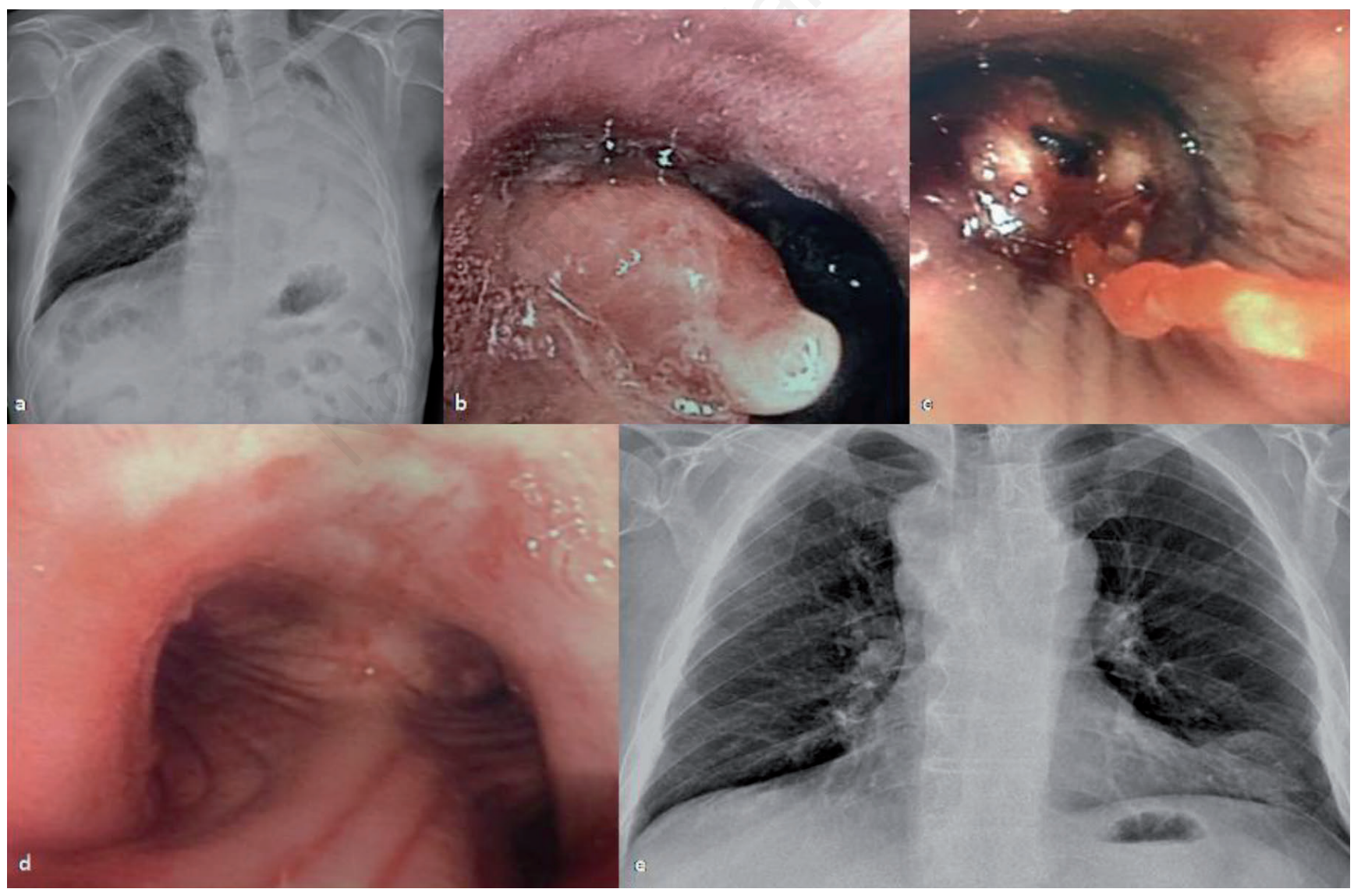

Figure 2. a) Chest X-ray at admission showed atelectasis of the left lung. b) Polypoid endobronchial vegetation extending at bridge between left and right main bronchus. c) Endoluminal vegetation in the distal left main bronchus. d) Complete airway patency was obtained after rigid bronchoscopic tumour resection. e) Chest X-ray post rigid bronchoscopy procedure. 


\section{Discussion}

Lower socioeconomic status has been accepted to be linked to worse chronic health outcomes independently to severity of disease. Cultural factors can influence the communication between physicians and patient resulting in underestimated symptom intensities and a low alarm of life-threatening status [1-2].

Malignant central airway obstruction represents a silent and progressive airway obstruction for $20-30 \%$ of patients with primary lung cancer until to result in life-threatening disease [3].

The misperception of dyspnoea can lead the delayed seeking of medical care and increasing access to intensive care if it is not correctly evaluated.

Endotracheal intubation represents the "gold standard" for securing airway in patient with malignant airway obstruction although definitive airway control can be re-established with urgent rigid bronchoscopy. Rigid bronchoscopy intervention with mechanical debulking represents the main procedure to rapidly securing airway patency in patient with life-threatening dyspnoea from central airways obstruction.

In our cases, the impact of emergency rigid bronchoscopy on acute ventilator distress was technically successful, although withdrawal from mechanical ventilation was different from each other. This date reflects the pulmonary functional status as independent risk factors for mortality in lung cancer patients undergone prolonged mechanical ventilation [4]. Multiple variables as weakness of the respiratory muscle, reduced compliance of the chest wall and the loss of the elastic properties of the lung can influence the weaning from mechanical ventilation making useless endoscopic airway recanalization [5].

\section{Conclusions}

In this case report, two lower social class patients underwent emergency rigid bronchoscopy for acute malignant airway obstruction have been reported. Despite the best management different outcome was observed and the following conclusions will be observed:

Lower social class can affect the communication between physicians and patient resulting in underestimated symptom intensities;

Acute respiratory failure can be a thunderbolt in malignant airway obstruction;

Emergency rigid bronchoscopy can restore airway patency in patient with life-threatening dyspnoea from central airways obstruction but it can affect the outcome in patient undergoing mechanical ventilation.

\section{References}

1. Buchanan D, Milroy D, Baker L, et al. Perceptions of anxiety in lung cancer patients and their support network. Support Care Cancer 2010;18:29-36.

2. Willems S, De Maesschalck S, Deveugele M, et al.. Socio-economic status of the patient and doctor patient communication: does it make a difference? Patient Educ Couns 2005;56:139-46.

3. Colt HG, Harrell JH. Therapeutic rigid bronchoscopy allows level of care changes in patients with acute respiratory failure from central airways obstruction. Chest 1997;112:202-6.

4. Stanopoulos IT, Beamis JF, Marinez FJ, et al. Laser bronchoscopy in respiratory failure from malignant airway obstruction. Crit Care Med1993;21:386-91.

5. Laghi F, Tobin MJ. Disorders of the respiratory muscles. Am J Respir Crit Care Med 2003;168:10-48. 